\title{
Commemoration of Angelo Cassatella
}

\author{
Franco Giovannelli** \\ INAF - Istituto di Astrofisica e Planetologia Spaziali, Via del Fosso del Cavaliere, 100, 00133 \\ Roma, Italy \\ E-mail: Eranco.qiovannellidiaps.inaf.it
}

In this short paper I celebrate the life of Angelo Cassatella. Angelo was one of my colleagues among those who are scattered everywhere! For this particular "strange" case of our connection, though he was in my own old institute (Laboratorio di Astrofisica Spaziale, CNR), we really knew each other since 1980 in the historical ESA-VILSPA center (Villafranca del Castillo, Spain), where he was resident astronomer and I was a frequent visitor and user of the IUE (International Ultraviolet Explorer) via that site. This brief remembrance does not list the many important scientific contributions of Angelo, but simply paints his humanity through a few episodes of daily life that will stay in my mind forever.

The Golden Age of Cataclysmic Variables and Related Objects - III, Golden2015

7-12 September 2015

Palermo, Italy

* Speaker.

${ }^{\dagger}$ A footnote may follow. 


\section{In memoriam}

Angelo was a good scientist, but above all a peaceful, kind and courteous man.

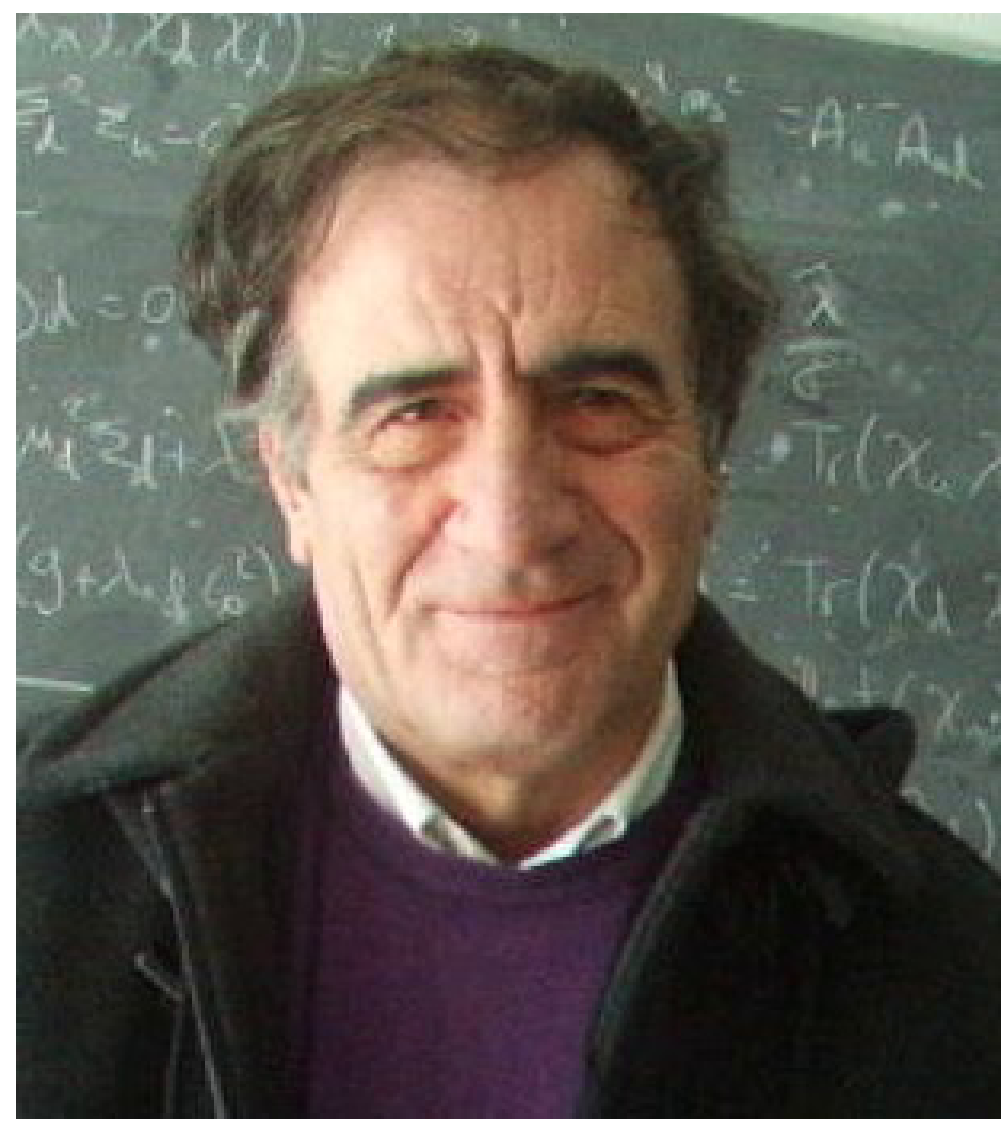

Figure 1: Angelo Cassatella (12th March 1944 - 29th June 2015).

We shared so many IUE shifts in VILSPA that it is difficult to remember all of them. During one of those visits, I had a problem with my health due to a sudden increase of glycemia. Angelo provided assistance to me and helped me to find a proper doctor. This might appear as natural, but in fact what struck me was the speed and the solidarity with which Angelo took care of me. This event will stay in my mind forever.

Here, I want to tell you a story that can paint our relationship in a mixture of science and daily life. Because of my frequent stays in Madrid as an IUE guest observer, Angelo invited me to be a member of the Local Organizing Committee of the IAU Colloquium, N. 122 on "Physics of Classical Novae", which was held in Madrid on 27-30 June 1989. I accepted with great enthusiasm. One of my "key" tasks was to verify the quality of the restaurant which was booked for the official banquet, and in particular to control the quality of the wines that would be served at the banquet. To that end, I chose elegant attire, and together with a group of friends we went to the restaurant for a dinner without announcing myself as the controller of the official menu chosen for the banquet.

During the dinner, my friends were joking about my "hard job", and many of them, especially the women, offered to be my assistant for this "terrible" burden. I answered seriously that it was 
"my job" for which I had received the assignment by Angelo Cassatella, the Boss! At the end of the dinner I introduced myself as controller and together with the director of the restaurant, we changed the wines to be served during the official banquet. The banquet was great, as well as the scientific colloquium for which Angelo and Roberto Viotti were the editors of the proceedings.

Angelo's interests were not only scientific. He successfully cultivated a passion for classical music, playing the violin very well.

Within the Spanish National Project, "A University, A Universe", he worked to make our Universe accessible to citizens at large. Angelo gave his availability because he was convinced that it is necessary for us to contribute in order to overcome the current existing social dichotomy between "Sciences" and "Humanities". In this context Angelo performed as a violinist in the ConcertConference "Music to the Universe", held at the "Teatro Apolo" in Almeria on June 17, 2009, which was organized by the University of Almeria (Andalucia, Spain). In this effort, he performed together with Telmo Fernández (guitar) and Serguei Mesropian (cello) with texts by John Beckman (our famous astrophysicist colleague and friend), and with astronomical images from ESA, the European Space Agency.

Angelo's life was strongly marked by his long stay in VILSPA - so much so that he married with Carmen, a beautiful Spanish woman, who bore him two sons: Pablo and Juan.

I would like to remember Angelo as a fisherman of stars, using the beautiful and innocent image produced by a little girl seven years old.

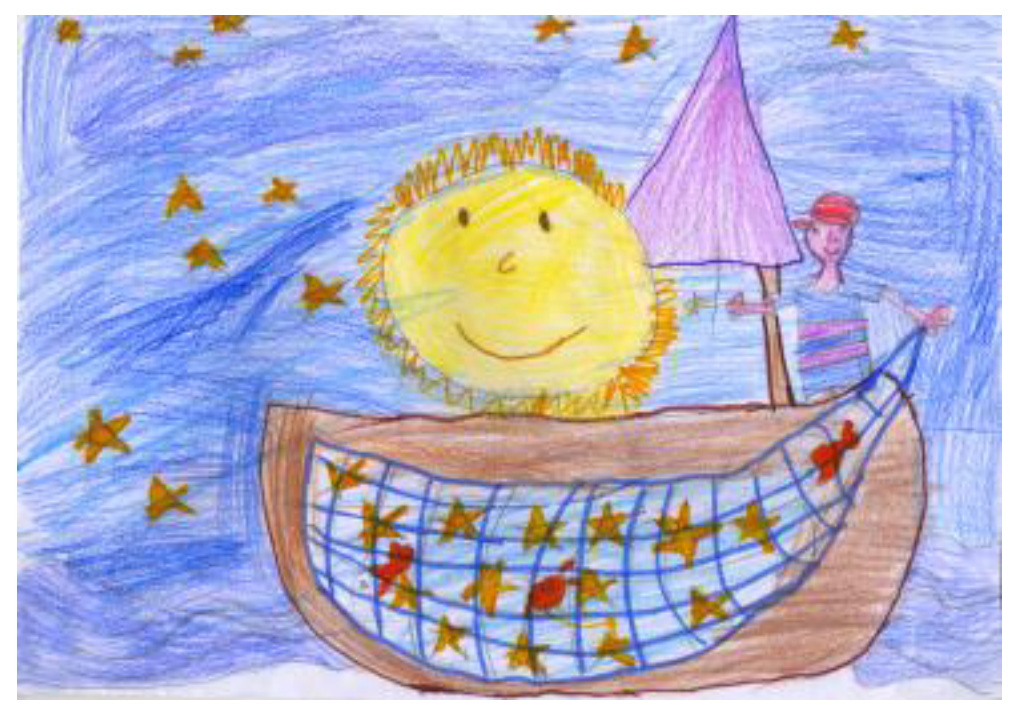

Figure 2: Angelo: the fisherman of stars (credit: Anais, G.)

\section{Angelo, rest in peace among the stars!}

